\title{
Practical Tips
}

\section{Coding for Smoking and Tobacco-Use Cessation Counseling}

Beginning March 2005, Medicare Part B began providing coverage for smoking and tobacco-use cessation counseling. Here are some of the most frequently asked questions about billing for this service.

\section{Are all Medicare beneficiaries eligible for smoking cessation counseling?}

No. Medicare statute states that coverage is limited to Medicare beneficiaries who use tobacco and have a disease or adverse health effect found by the US Surgeon General to be linked to tobacco use. In addition, it is limited to those taking certain therapeutic agents whose metabolism or dosage is affected by tobacco use as based on FDA-approved information. Additionally, patients must be competent and alert at the time that services are provided.

What codes are used to bill for these services?

Two new codes were added to the Health Care Financing Administration's Common Procedure Coding System:

- G0375-Smoking and tobacco-use cessation counseling visit; intermediate session of more than 3 minutes and up to 10 minutes

- G0376 - Smoking and tobacco-use cessation counseling visit; intensive session of more than 10 minutes

Smoking and tobacco-use cessation counseling services of 3 minutes or less are bundled into the evaluation and management $(\mathrm{E} \& \mathrm{M})$ service.

\section{Who can provide these services?}

Physicians and qualified nonphysician practitioners can bill Medicare for smoking and tobacco-use cessation counseling.

\section{How often can these codes be used?}

Medicare Part B covers two smoking cessation attempts each year. Each attempt may include a maximum of four intermediate or intensive sessions. A total of eight sessions are covered in a 12 -month period.

\section{Is special documentation required?}

Claims for smoking and tobacco-use cessation counseling should be submitted with a diagnosis code that reflects the condition the patient has that is adversely affected by tobacco use or the condition the patient is being treated for with a therapeutic agent whose metabolism or dosing is affected by tobacco use. Providers should include appropriate documentation in the patient record to adequately demonstrate that Medicare coverage conditions were met for any services provided.

\section{Can an ESM service be provided on the same day as} smoking and tobacco-use cessation counseling?

Yes. If an E\&M service is provided on the same day as smoking and tobacco-use cessation counseling, providers should choose the appropriate E\&M code and use modifier 25 to show that the E\&M service is a separately identifiable service from the smoking and tobacco-use cessation counseling service.

Can these codes be used for payers other than Medicare? Codes G0375 and G0376, and the associated reimbursement policies, were developed specifically for Medicare patients. Other payers may choose to recognize these codes for nonMedicare patients, but they are not required to do so. Check with your payers for their policy on coverage and reimbursement for smoking and tobacco-use cessation counseling.

\section{Diagnosis Coding Update}

Changes in the 2007 ICD-9-CM (The International Classification of Diseases, Ninth Revision, Clinical Modification) manual include new codes, revisions, and deleted codes frequently used by oncology and hematology practices. Table 1 summarizes many of these changes. For a complete list, refer to the 2007 ICD-9-CM manual. Changes in ICD-9-CM were effective October 1, 2006.

DOI: 10.1200/JOP.0712502 
Table 1. Updates to Diagnosis Coding

\section{Code}

New codes

238.71

238.72

238.73

238.74

238.75

238.76

238.79

284.01

284.09

284.1

284.2

288.00

288.01

288.02

288.03

288.04

288.09

288.4

288.50

288.51

288.59

288.60

288.61

288.62

288.63

288.64

288.65

288.69

289.53

289.83

338.3

528.01

795.81

795.82

795.89

V86.0

V86.1

Revised codes

285.29

790.93

Invalid codes

238.7

277.3

284.0

288.0

\section{Diagnosis}

Essential thrombocythemia

Low-grade myelodysplastic syndrome lesions

High-grade myelodysplastic syndrome lesions

Myelodysplastic syndrome with $5 q$ deletion

Myelodysplastic syndrome, unspecified

Myelofibrosis with myeloid metaplasia

Other lymphatic and hematopoietic tissues

Constitutional red blood cell aplasia

Other constitutional aplastic anemia

Pancytopenia

Myelophthisis

Neutropenia, unspecified

Congenital neutropenia

Cyclic neutropenia

Drug-induced neutropenia

Neutropenia due to infection

Other neutropenia

Hemophagocytic syndromes

Leukocytopenia, unspecified

Lymphocytopenia

Other decreased white blood cell count

Leukocytosis, unspecified

Lymphocytosis (symptomatic)

Leukemoid reaction

Monocytosis (symptomatic)

Plasmacytosis

Basophilia

Other elevated white blood cell count

Neutropenic splenomegaly

Myelofibrosis

Neoplasm related pain (acute) (chronic)

Mucositis (ulcerative) due to antineoplastic therapy

Elevated carcinoembryonic antigen (CEA)

Elevated cancer antigen 125 (CA 125)

Other abnormal tumor markers

Estrogen receptor-positive status (ER+)

Estrogen receptor-negative status (ER-)

Anemia of other chronic disease

Elevated prostate specific antigen

Other lymphatic and hematopoietic tissues

Amyloidosis

Constitutional aplastic anemia

Agranulocytosis 